\title{
Solitons in nonlocal nonlinear media: Exact solutions
}

\section{Krolikowski, Wieslaw; Bang, Ole}

\section{Published in:}

Physical Review E. Statistical, Nonlinear, and Soft Matter Physics

Link to article, DOI:

10.1103/PhysRevE.63.016610

Publication date:

2001

\section{Document Version}

Publisher's PDF, also known as Version of record

Link back to DTU Orbit

\section{Citation (APA):}

Krolikowski, W., \& Bang, O. (2001). Solitons in nonlocal nonlinear media: Exact solutions. Physical Review E. Statistical, Nonlinear, and Soft Matter Physics, 63(1), 016610. https://doi.org/10.1103/PhysRevE.63.016610

\section{General rights}

Copyright and moral rights for the publications made accessible in the public portal are retained by the authors and/or other copyright owners and it is a condition of accessing publications that users recognise and abide by the legal requirements associated with these rights.

- Users may download and print one copy of any publication from the public portal for the purpose of private study or research.

- You may not further distribute the material or use it for any profit-making activity or commercial gain

- You may freely distribute the URL identifying the publication in the public portal

If you believe that this document breaches copyright please contact us providing details, and we will remove access to the work immediately and investigate your claim 


\title{
Solitons in nonlocal nonlinear media: Exact solutions
}

\author{
Wiesław Królikowski \\ Australian Photonics Cooperative Research Centre, Laser Physics Centre, Research School of Physical Science and Engineering, \\ The Australian National University, Canberra ACT 0200, Australia \\ Ole Bang \\ Department of Mathematical Modelling, Building 305/321, Technical University of Denmark, DK-2800 Kgs. Lyngby, Denmark
}

(Received 31 May 2000; published 22 December 2000)

\begin{abstract}
We investigate the propagation of one-dimensional bright and dark spatial solitons in a nonlocal Kerr-like media, in which the nonlocality is of general form. We find an exact analytical solution to the nonlinear propagation equation in the case of weak nonlocality. We study the properties of these solitons and show their stability.
\end{abstract}

DOI: 10.1103/PhysRevE.63.016610

PACS number(s): 42.65.Tg

\section{INTRODUCTION}

Let us consider a phenomenological model of nonlocal nonlinear Kerr-type media, in which the refractive index change $\Delta n$ induced by a beam with intensity $I(x, z)$ can be represented in general form as

$$
\Delta n(I)= \pm \int_{-\infty}^{\infty} R\left(x^{\prime}-x\right) I\left(x^{\prime}, z\right) d x^{\prime}
$$

where the positive (negative) sign corresponds to a focusing (defocusing) nonlinearity and $x$ and $z$ denote transverse and propagation coordinates, respectively. The real, localized, and symmetric function $R(x)$ is the response function of the nonlocal medium, whose width determines the degree of nonlocality. For a singular response, $R(x)=\delta(x)$, the refractive index change becomes a local function of the light intensity, $\Delta n(I)= \pm I(x, z)$, i.e., the refractive index change at a given point is solely determined by the light intensity at that very point. With increasing width of $R(x)$ the light intensity in the vicinity of the point $x$ also contributes to the index change at that point. In the limit of a highly nonlocal response Snyder and Mitchell showed that the beam evolution was described by the simple equation for a linear harmonic oscillator [1]. The influence of nonlocality of the nonlinear response on the dynamics of beams was illustrated for the special logarithmic nonlinearity, which allows exact analytical treatment [2].

While Eq. (1) is a phenomenological model, it nevertheless describes several real physical situations. Possible physical mechanisms responsible for this type of nonlinear response includes various transport effects, such as heat conduction in materials with thermal nonlinearity [3-5], diffusion of molecules or atoms accompanying nonlinear light propagation in atomic vapors [6], and drift and/or diffusion of photoexcited charges in photorefractive materials $[7,8]$. A highly nonlocal nonlinearity of the form (1) has also been identified in plasmas [9-13] and it appears as a result of many-body interaction processes in the description of BoseEinstein condensates [14].

Even though it is quite apparent in some physical situations that the nonlinear response in general is nonlocal (as in the case of thermal lensing), the nonlocal contribution to the refractive index change was often neglected $[15,16]$. This is justified if the spatial scale of the beam is large compared to the characteristic response length of the medium (given by the width of the response function). However, for very narrow beams the nonlocality can be of crucial importance and has to be taken into account. For instance, it has been shown theoretically that a weak nonlocal contribution arrests collapse (catastrophic self-focusing) of high power optical beams in a self-focusing medium and leads to the formation of stable two-dimensional (2D) (diffracting in two transverse dimensions) solitons $[12,13,17,18]$. On the other hand, a purely nonlocal nonlinearity leads to formation of so-called cusp solitons, which, however, are unstable [9].

Some of the consequences of nonlocality in the nonlinear response have been observed experimentally. Suter and Blasberg reported stabilization of 2D solitary beams in atomic vapors due to atomic diffusion, which carries excitation away from the interaction region [6]. Also, the discrepancy between the theoretical model of dark solitons and that observed experimentally in a medium with thermal nonlinearity has been associated with nonlocality of the nonlinearity $[15,16]$.

Here, we investigate the propagation of one-dimensional (1D) beams in nonlinear media having a weakly nonlocal response of the general form (1). Our goal is to find exact analytical solutions for bright and dark spatial solitons and use them to determine soliton properties, such as existence and stability. We start with the paraxial wave equation describing propagation of a 1D beam with envelope function $\psi=\psi(x, z)$ and intensity $I=I(x, z)=|\psi(x, z)|^{2}$,

$$
i \partial_{z} \psi+\frac{1}{2} \partial_{x}^{2} \psi+\Delta n(I) \psi=0
$$

When the nonlocality is weak, i.e., when the response function $R(x)$ is narrow compared to the extent of the beam (see Fig. 1), we can expand $I\left(x^{\prime}, z\right)$ around the point $x^{\prime}=x$ to obtain

$$
\Delta n(I)= \pm\left(I+\gamma \partial_{x}^{2} I\right)
$$

where the nonlocality parameter $\gamma>0$ is given by 


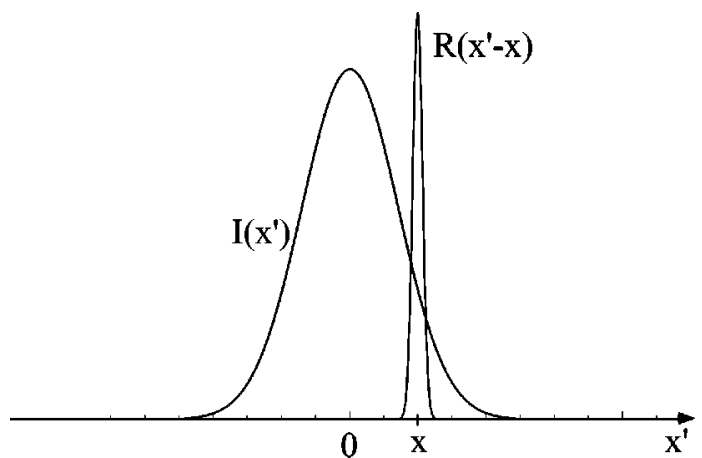

FIG. 1. Intensity profile $I\left(x^{\prime}\right)=I\left(x^{\prime}, z\right)$ and response function $R\left(x^{\prime}-x\right)$ in the weakly nonlocal limit.

$$
\gamma=\frac{1}{2} \int_{-\infty}^{\infty} R(x) x^{2} d x
$$

and where we have assumed that the response function is normalized, $\int_{-\infty}^{\infty} R(x) d x=1$. Note that for $R(x)=\delta(x), \gamma$ $=0$ and Eq. (3) describes the local Kerr nonlinearity. For weakly nonlocal meda $\gamma \ll 1$ is a small parameter.

Substituting $\Delta n(I)$, given by Eq. (3), into Eq. (2) gives the modified nonlinear Schrödinger equation

$$
i \partial_{z} \psi+\frac{1}{2} \partial_{x}^{2} \psi \pm\left(|\psi|^{2}+\gamma \partial_{x}^{2}|\psi|^{2}\right) \psi=0 .
$$

The weak nonlocality appears thus as a perturbation to the local nonlinear refractive index change. For a single peak beam in a self-focusing medium this perturbation is of negative sign in the central part of the beam, where it serves to decrease the refractive index change. Hence, even for very narrow and sharp intensity distributions, the resulting selfinduced waveguide will be wide and a smooth function of the transverse coordinates. In some sense, this is similar to saturation of the nonlinearity. One may therefore expect that certain features of solitons of Eq. (5) will be reminiscent of those exhibited by solitons in saturable nonlinear media [19]. It transpires, however, that nonlocality also results in new effects, especially for self-defocusing nonlinearities. In the following we consider separately the case of self-focusing and self-defocusing nonlinearities.

\section{BRIGHT SOLITONS}

For a self-focusing nonlinearity the sign of the refractive index change is positive. We search for a stationary bright soliton solution to Eq. (5) of the form

$$
\psi(x, z)=u(x) \exp (i \Gamma z)
$$

where the profile $u=u(x)$ is real, symmetric, and exponentially localized and the propagation constant $\Gamma>0$ is positive. For this solution Eq. (5) reduces to

$$
\partial_{x}^{2} u+2\left(u^{2}-\Gamma\right) u+2 \gamma u \partial_{x}^{2} u^{2}=0,
$$

which can be integrated once to give the equation

$$
\left(1+4 \gamma u^{2}\right)\left(\partial_{x} u\right)^{2}+\left(u^{2}-2 \Gamma\right) u^{2}=C,
$$

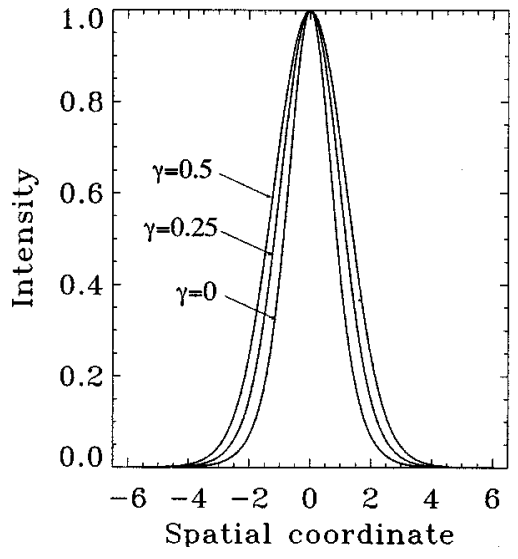

FIG. 2. Intensity profiles of bright solitons with unit amplitudes $\rho_{0}=1$ for different degrees of nonlocality $\gamma$.

where $C$ is an integration constant. For exponentially localized solutions $C=0$. Using that $u(x)$ has its maximum $u_{0}$ at the center $x=0$ we further obtain the well-known relation between the propagation constant $\Gamma$ and the amplitude $u_{0}$

$$
u_{0}^{2}=2 \Gamma \text {. }
$$

Equation (8) can therefore be simplified to

$$
\left(\partial_{x} u\right)^{2}=u^{2}\left(u_{0}^{2}-u^{2}\right) /\left(1+4 \gamma u^{2}\right) .
$$

Interestingly, had the local and nonlocal contributions been of opposite signs, i.e., $\gamma<0$ (as can happen for intense laser beams in plasmas), then a solution to Eq. (10) would only exist if the peak intensity $\rho_{0}=u_{0}^{2}$ is less than the critical value $\rho_{\mathrm{cr}}=1 /|4 \gamma|$ (see also [11]). A final integration leads to

$$
\pm x=\frac{1}{u_{0}} \tanh ^{-1}\left(\frac{\sigma}{u_{0}}\right)+\sqrt{4 \gamma} \tan ^{-1}(\sqrt{4 \gamma} \sigma),
$$

where we have defined the intensity $\rho=u^{2}$ and the normalized intensity $\sigma^{2}=\left(\rho_{0}-\rho\right) /(1+4 \gamma \rho)$. This implicit relation gives the profile of bright spatial solitons propagating in weakly nonlocal Kerr-like media. In the local limit, $\gamma=0$, we recover from Eq. (11) the well-known profile $u(x)$ $=u_{0} \operatorname{sech}\left(u_{0} x\right)$ of the $1 \mathrm{D}$ bright soliton in a Kerr medium.

In Fig. 2 we show the intensity profile of the solution (11) for different values of the nonlocality parameter $\gamma$. Evidently, an increase in the degree of nonlocality results in an increase of the soliton width-nonlocality smooths out the refractive index profile thereby leading to a broadening of the beam. This effect is more clearly seen in Fig. 3 where we plot the width of the bright solitons (full width at half maximum of the intensity profile) versus the degree of nonlocality $\gamma$ for different peak intensities; the soliton width increases monotonically with the degree of nonlocality.

An important aspect of any family of soliton solutions is their stability properties. For single-peak solitons stability is determined by the dependence of the power

$$
P=\int_{-\infty}^{\infty}|\psi(x, z)|^{2} d x
$$




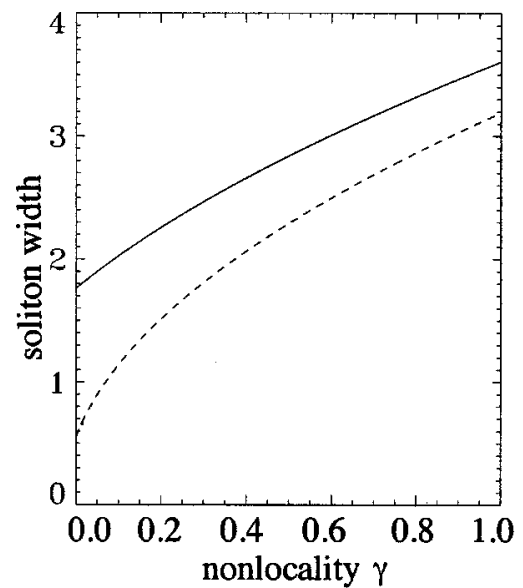

FIG. 3. Soliton width versus degree of nonlocality $\gamma$ for peak intensities $\rho_{0}=1$ (solid) and $\rho_{0}=10$ (dashed).

on the propagation constant $\Gamma$. Solitons are stable if $d P / d \Gamma>0$ and unstable otherwise [20]. For the model considered here $P(\Gamma)$ can be found analytically to

$$
P=\sqrt{\rho_{0}}+\frac{1+4 \gamma \rho_{0}}{\sqrt{4 \gamma}} \tan ^{-1}\left(\sqrt{4 \gamma \rho_{0}}\right)
$$

by using Eq. (10). For weakly nonlocal media with $\gamma \ll 1$ the power is approximately given by

$$
P=P_{0}\left(1+\frac{4}{3} \gamma \rho_{0}-\frac{16}{15} \gamma^{2} \rho_{0}^{2}+\cdots\right),
$$

where $P_{0}=2 \sqrt{\rho_{0}}$ is the soliton power in the limit of a local response with $\gamma=0$. The derivative $d P / d \Gamma$ can easily be found from Eq. (13) and it transpires that the power is a monotonically increasing function of the propagation constant (for $\gamma>0$ ), indicating that the solitons are stable.

Interestingly again, had $\gamma$ been negative, then the bright solitons would exist for sufficiently low-peak intensities, $\rho_{0}$ $<1 /|4 \gamma|$, but be unstable for $\rho_{0}>0.7 /|4 \gamma|$, for which $d P / d \Gamma$ is negative (see again [11]).

To demonstrate the stability of the bright solitons for $\gamma$ $>0$ we numerically integrated Eq. (5) using the split-step Fourier method and the exact soliton solution as an initial condition. In all simulations (for both bright and dark solitons) we used a steplength of $d z=0.001$ and a transverse resolution of $d x=0.05$. Results of the numerical simulations are shown in Fig. 4 where we demonstrate propagation and collision of two bright solitons with unit amplitude $\rho_{0}=1$. It is evident that the solitons propagate in a stable manner. The collision, on the other hand, is not completely elastic and causes the soliton amplitude and width to oscillate slightly.

The bright solitons of the nonlocal nonlinear Schrödinger Eq. (5) were considered by Davydova and Fischuk in the context of focusing of upper hybrid waves in plasma [13]. In particular, the existence of bright soliton solutions and their stability was reported by these authors. However, the explicit expression (11) was not given and no numerical confirmation was presented.

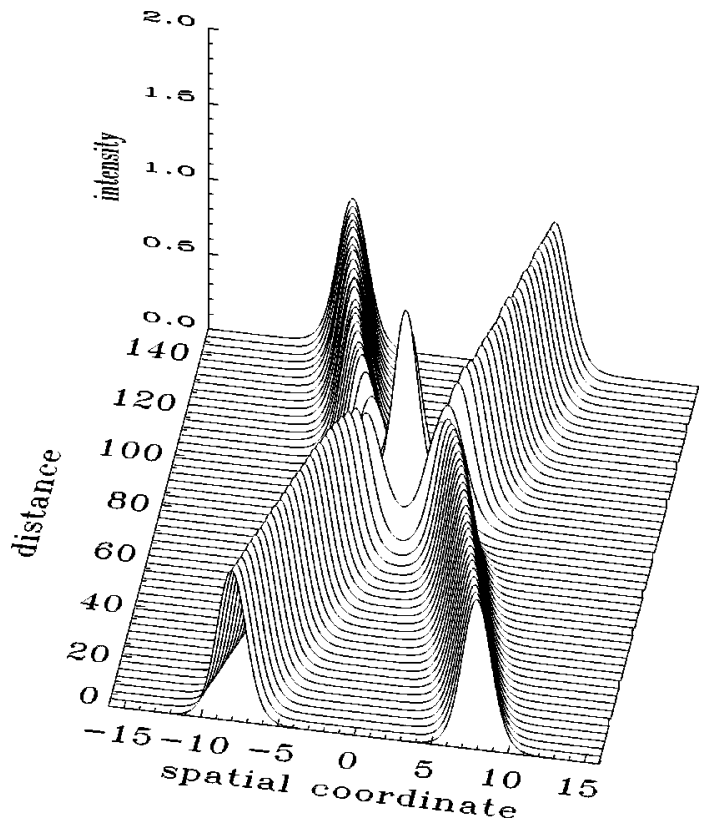

FIG. 4. Collision of unit amplitude bright solitons in a weakly nonlocal Kerr-like medium with $\gamma=0.1$.

\section{DARK SOLITONS}

We now consider the impact of weak nonlocality in the case of a self-defocusing nonlinearity, which corresponds to a negative sign in Eq. (3). We introduce a spatial variable $\zeta=x-V z$, with $V$ being the soliton transverse velocity, and look for stationary solutions of the form

$$
\psi(x, z)=\sqrt{\rho(\zeta)} \exp [i \Gamma z+i \phi(\zeta)]
$$

where $\rho(\zeta)$ is the soliton intensity and $\phi(\zeta)$ its phase. Substitution of this form into Eq. (5) yields

$$
\begin{gathered}
\partial_{\zeta}\left[\rho\left(\partial_{\zeta} \phi-V\right)\right]=0, \\
2 \rho(1-4 \gamma \rho) \partial_{\zeta}^{2} \rho-\left(\partial_{\zeta} \rho\right)^{2}-4 \rho^{2}\left(\partial_{\zeta} \phi\right)^{2}+8 V \rho^{2} \partial_{\zeta} \phi \\
-8 \rho^{2}(\Gamma+\rho)=0 .
\end{gathered}
$$

We are interested in dark solitons, i.e., solutions with an intensity dip on a constant background. Integrating the system (16),(17) once we obtain that the soliton background intensity $\rho_{0}=u_{0}^{2}$ determines the soliton propagation constant $\Gamma$ and that the center intensity $\rho_{1}=u_{1}^{2}$ determines the transverse velocity $V$, through the relations

$$
V^{2}=\rho_{1}, \quad \Gamma=-\rho_{0},
$$

which are exactly the same as those obtained for a purely local response. We further find that the soliton intensity $\rho(\zeta)$ is governed by the equation

$$
\left(\partial_{\zeta} \rho\right)^{2}=4\left(\rho-\rho_{1}\right)\left(\rho_{0}-\rho\right)^{2} /(1-4 \gamma \rho) .
$$

Obviously, a solution to Eq. (19) exists only if the background intensity does not exceed a certain critical value, 


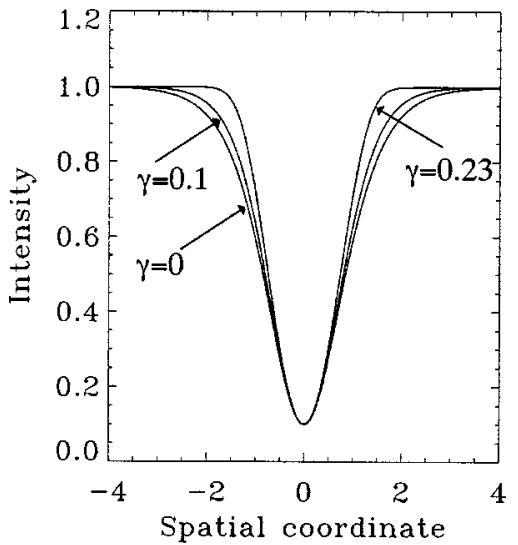

FIG. 5. Intensity profiles of dark solitons with $\rho_{0}=1$ and $\rho_{1}$ $=0.1$ for different values of the nonlocality parameter $\gamma$.

$$
\rho_{0}<\rho_{\mathrm{cr}}=1 /|4 \gamma|
$$

which coincides with the critical peak intensity above which bright solitons does not exist in a focusing nonlocal medium when $\gamma<0$, as discussed above.

It can be shown that the relation (20) represents the stability condition for the plane wave solution to Eq. (5) with a defocusing nonlinearity. For background intensities larger than $\rho_{\mathrm{cr}}$ the plane-wave solutions become modulationally unstable [21].

Integrating Eq. (19) once more we obtain

$$
\pm \zeta=\frac{1}{\delta_{0}} \tanh ^{-1}\left(\frac{\delta}{\delta_{0}}\right)+\sqrt{4 \gamma} \tan ^{-1}(\sqrt{4 \gamma} \delta)
$$

which is an implicit relation between the soliton intensity $\rho$ and the spatial coordinate $\zeta$, with the normalized intensity now given by $\delta^{2}(\rho)=\left(\rho-\rho_{1}\right) /(1-4 \gamma \rho)$ and $\delta_{0}=\delta\left(\rho_{0}\right)$. For the soliton phase we obtain

$$
\pm \phi=\tan ^{-1}\left(\frac{\delta}{u_{1}}\right)-u_{1} \sqrt{4 \gamma} \tan ^{-1}(\sqrt{4 \gamma} \delta)
$$

where we have used the gauge invariance of Eq. (2) to remove a constant phase contribution (phase in the center). In Fig. 5 we present examples of the intensity profile of the dark solitons for different values of the nonlocality parameter $\gamma$. These graphs show that increasing nonlocality decreases the width of the soliton. To better illustrate this effect we plot in Fig. 6 the width of the dark solitons (defined as the distance between points where the intensity is $\left(\rho_{0}\right.$ $\left.+\rho_{1}\right) / 2$ ) versus $\gamma$ for several values of the soliton contrast $a=\left(\rho_{0}-\rho_{1}\right) / \rho_{0}$.

We see that unlike saturation, which tends to increase the width of dark solitons, nonlocality has the opposite effect. This is due to the fact that nonlocality causes the nonlinear index change to advance towards regions of lower light intensity. In the case of bright solitons this effect leads to soliton expansion. The plots in Fig. 6 also indicate the interesting effect that for strong nonlocality, all dark solitons acquire the same width. Actually, in this limit, i.e., when $\gamma$ ap-

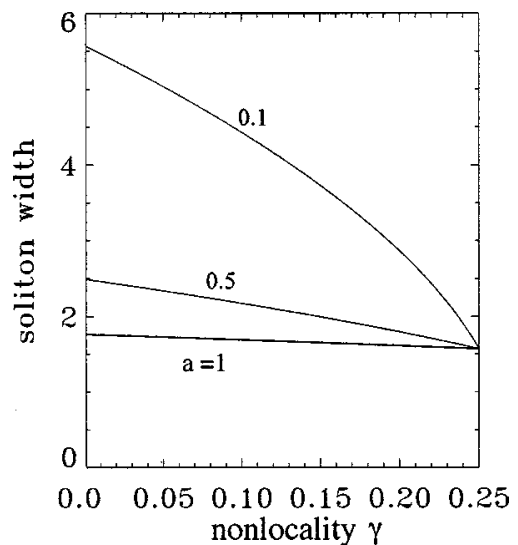

FIG. 6. Width of dark solitons versus degree of nonlocality $\gamma$ for $\rho_{0}=1$ and different contrasts $a=\left(\rho_{0}-\rho_{1}\right) / \rho_{0}$.

proaches the critical value $\gamma=1 /\left(4 \rho_{0}\right)$ the relation (21) can be inverted leading to a simple expression for the soliton intensity profile

$$
\rho= \begin{cases}\rho_{0}\left[1-a \cos ^{2}\left(u_{0} \zeta\right)\right], & \left|u_{0} \zeta\right| \leqslant \pi / 2 \\ \rho_{0}, & \left|u_{0} \zeta\right| \geqslant \pi / 2 .\end{cases}
$$

The solution (23) confirms that in this limit all dark solitons have the same width $\zeta_{\mathrm{cr}}=\pi /\left(2 u_{0}\right)$ independent of the contrast.

With a nontrivial phase structure, these dark solitons can be represented in the complex plane describing the real and imaginary part of the soliton amplitude $\psi(\zeta)=\sqrt{\rho} \exp (i \phi)$. Any soliton solution is then represented by a trajectory in this plane. Figure 7 shows such a phase diagram $(\gamma=0.1)$ for several values of the soliton contrast. The circle represents the background intensity $\rho_{0}=1$ and nonlocal soliton solutions are plotted using solid lines. The dashed line corresponds to the same contrast soliton solution in the purely local case $(\gamma=0)$. The total phase change across the soliton is given by the angle subtended by two lines connecting the origin with points of intersection of the soliton curve and the circle. One can see directly from Fig. 7 that the total phase change across the soliton in a nonlocal medium is always

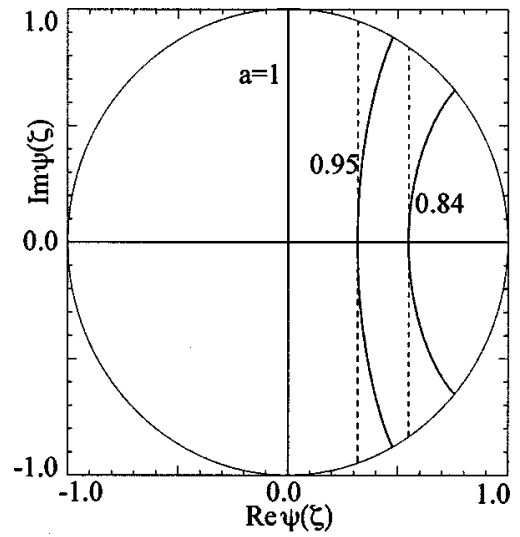

FIG. 7. Real and imaginary part of dark solitons with $\rho_{0}=1$ in a weakly nonlocal medium with $\gamma=0.1$. 


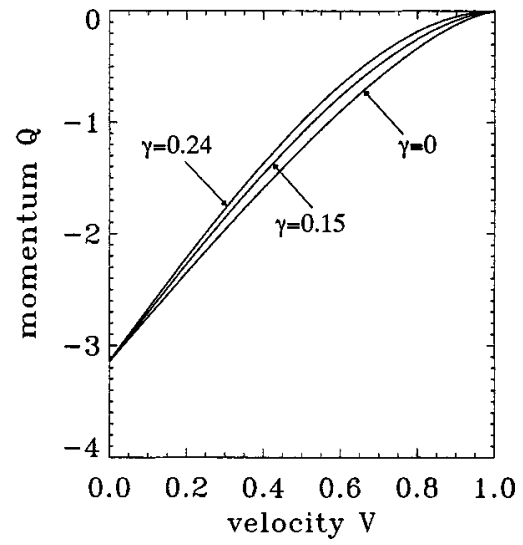

FIG. 8. Momentum $Q$ of dark solitons with $\rho_{0}=1$ versus velocity $V$ for different degrees of nonlocality, $\gamma$.

less than in the local case. Furthermore, the soliton phase profile closely resembles that of a threshold nonlinearity [22].

The linear stability of dark solitons can be determined by considering the conserved quantities of the nonlinear Schrödinger Eq. (5) with defocusing nonlinearity, namely the renormalized Hamiltonian

$$
H=\frac{1}{2} \int_{-\infty}^{\infty}\left[\left|\partial_{x} \psi\right|^{2}+\left(|\psi|^{2}-\rho_{0}\right)^{2}-\gamma\left(\partial_{x}|\psi|^{2}\right)^{2}\right] d x
$$

and the renormalized momentum

$$
Q=\frac{i}{2} \int_{-\infty}^{\infty}\left(\psi \partial_{x} \psi^{*}-\psi^{*} \partial_{x} \psi\right)\left(1-\frac{\rho_{0}}{|\psi|^{2}}\right) d x
$$

A soliton solution propagating with velocity $V$ corresponds to an extremum of the Hamiltonian for fixed momentum, $\delta(H-V Q)=0$. It has been shown that the stability criterion for dark solitons is determined by the dependence of the momentum on the velocity, $Q=Q(V)$ [23]. For dark solitons to be stable the derivative of the momentum with respect to the velocity must be positive,

$$
d Q / d V>0 .
$$

In the case of a nonlocal nonlinearity the expression for the momentum can be evaluated analytically to

$$
\begin{aligned}
Q= & -2 \rho_{o} \tan ^{-1}\left(\frac{\delta_{0}}{\sqrt{\rho_{1}}}\right)+\left(\rho_{0}-\rho_{1}\right)\left(\frac{\sqrt{\rho_{1}}}{\delta_{0}}\right) \\
& +\left[1+4 \gamma\left(2 \rho_{0}-\rho_{1}\right)\right] \sqrt{\frac{\rho_{1}}{4 \gamma}} \tan ^{-1}\left(\sqrt{4 \gamma} \delta_{0}\right) .
\end{aligned}
$$

The dependence of the momentum on the velocity of dark solitons with background intensity $\rho_{0}=1$ is shown in Fig. 8 for several degrees of nonlocality $\gamma$. The monotonic increase of this function indicates that the dark solitons are stable.

This conclusion is confirmed by direct numerical simulations of Eq. (5) with initial conditions given by the exact solution (21),(22). Figure 9 illustrates the propagation and

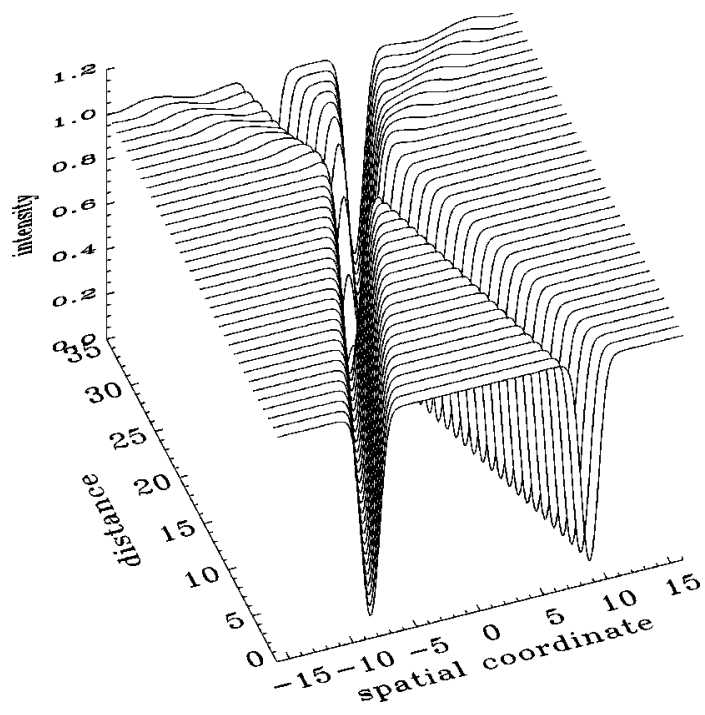

FIG. 9. Propagation and collision of identical dark solitons with $\rho_{0}=10 \rho_{1}=1$ in a weakly nonlocal medium with $\gamma=0.1$.

collision of two identical dark solitons with $\rho_{0}=10 \rho_{1}=1$ in a weakly nonlocal medium with $\gamma=0.1$. While the solitons propagate in a stable fashion, their collision is clearly inelastic with radiation being emitted from the impact area.

In conclusion, we have studied the properties of bright and dark one-dimensional spatial solitons in a general weakly nonlocal Kerr-like medium, in which the change in refractive index due to local and nonlocal contributions are of the same sign. We have found an exact analytical solution for both bright and dark solitons and used it to find the existence and stability regions of both solutions.

Stable bright soliton solutions were found to exist in focusing nonlocal media for all parameter values. Although they were previously predicted the solution was never explicitly written down. The effect of nonlocality is in some sense equivalent to that of saturation, to smooth out the index profile and thereby increase the soliton width.

Previously unknown dark soliton solutions were found to exist in defocusing nonlocal media for background intensities below a certain critical value, which correspond to the intensity at which the plane-wave solutions become unstable. We found that nonlocality leads to narrowing of the dark solitons, with all dark solitons acquiring the same width in the strongly nonlocal limit, independent of the soliton contrast. The total phase change across the dark soliton was found to be less than that in the purely local case, with the phase profile resembling that of a threshold nonlinearity.

Both nonlocal bright and dark spatial solitons appear to be stable under propagation. Preliminary studies of soliton collision revealed their inelastic character in analogy to collisions of solitons of nonintegrable models.

\section{ACKNOWLEDGMENTS}

O.B. acknowledges support from the Danish Technical Research Council under Talent Grant No. 9800400. 
[1] A. Snyder and J. Mitchell, Science 276, 1538 (1997).

[2] A. Snyder and J. Mitchell, J. Opt. Soc. Am. 16, 236 (1999).

[3] J.P. Gordon, R.C. Leite, R.S. Moore, S.P. Porto, and J.R. Whinnery, J. Appl. Phys. 36, 3 (1965).

[4] S. Akhmanov, D.P. Krindach, A.V. Migulin, A.P. Sukhorukov, and R.V. Khokhlov, IEEE J. Quantum Electron. 4, 568 (1968).

[5] M. Horovitz, R. Daisy, O. Werner, and B. Fischer, Opt. Lett. 17, 475 (1992); M.D. Iturbe-Castillo, J.J. Sanchez-Mondragon, and S. Stepanov, ibid. 21, 1622 (1996); D. Kip, E. Krätzig, V. Shandarov, and P. Moretti, ibid. 23, 343 (1998).

[6] D. Suter and T. Blasberg, Phys. Rev. A 48, 4583 (1993).

[7] S. Gatz and J. Herrmann, Opt. Lett. 23, 1176 (1998).

[8] B. Crosignani, A. Degasperis, E. DelRe, P. Di Porto, and A.J. Agranat, Phys. Rev. Lett. 82, 1664 (1999).

[9] M.V. Porkolab and M.V. Goldman, Phys. Fluids 19, 872 (1976).

[10] A.G. Litvak, V.A. Mironov, G.M. Fraiman, and A.D. Yunakovskii, Sov. J. Plasma Phys. 1, 31 (1975).

[11] A.G. Litvak and A.M. Sergeev, JETP Lett. 27, 517 (1978) [Pis'ma Zh. Éksp. Teor. Fiz. 27, 548 (1978)].

[12] H.L. Pecseli and J.J. Rasmussen, Plasma Phys. 22, 421 (1980).

[13] T.A. Davydova and A.I. Fishchuk, Ukr. J. Phys. 40, 487 (1995).
[14] F. Dalfovo, S. Giorgini, L.P. Pitaevskii, and S. Stringari, Rev. Mod. Phys. 71, 463 (1999); V.M. Perez-Garcia, V.V. Konotop, and J.J. Garcia-Ripoll, Phys. Rev. E 62, 4300 (2000).

[15] G.A. Swartzlander, Jr., and C.T. Law, Phys. Rev. Lett. 69, 2503 (1992).

[16] B. Luther-Davies and X. Yang, Opt. Lett. 17, 1755 (1992); A. Dreschuh, G.G. Paulus, F. Zacher, F. Grasbon, and H. Walther, Phys. Rev. E 60, 6111 (1999).

[17] S.K. Turitsyn, Teoreticheskaya i Matematicheskaya Fizika, 64, 226 (1985).

[18] S. Abe and A. Ogura, Phys. Rev. E 57, 6066 (1998).

[19] I. Bialynicki-Birula and J. Mycielski, Phys. Scr. 20, 539 (1979); S. Cowan et al., Can. J. Phys. 64, 311 (1986); S. Gatz and J. Herman, Opt. Lett. 17, 484 (1992); A. Snyder and A. Sheppard, ibid. 18, 482 (1983).

[20] E.W. Ladtke, K.H. Spatschek, and L. Stenflo, J. Math. Phys. 24, 2764 (1983).

[21] W. Krolikowski and O. Bang, Modulation Instability in Nonlocal Nonlinear Media (unpublished).

[22] W. Krolikowski, N. Akhmediev, and B. Luther-Davies, Phys. Rev. E 48, 3980 (1993).

[23] Y. Kivshar and W. Krolikowski, Opt. Lett. 20, 1527 (1995). 